\title{
Pulsed NMR Investigation on the Polymerization of Methyl Methacrylate
}

\author{
Takuzo KUROTU \\ Department of Chemistry, The National Defense Academy, \\ Yokosuka-shi, Kanagawa 239, Japan
}

(Received March 5, 1986)

\begin{abstract}
Nuclear magnetic resonance transversal relaxation time $\left(T_{2}\right)$ was measured as a function of polymerization time for methyl methacrylate at various temperatures. Three kinds of relaxation times, $T_{21}$ (long), $T_{2 \mathrm{~m}}$ (intermediate), and $T_{2 \mathrm{~s}}$ (short) are observed as the polymerization proceeds. $T_{21}$ decreases drastically at a certain reaction time, while $T_{2 s}$ changes only slightly throughout the process. The fraction of the $T_{21}$ component decreases abruptly when $T_{21}$ decreases, while that of the $T_{2 \mathrm{~s}}$ component increases. The former reflects the decrease in monomer concentration, whereas the latter corresponds to the entanglement of polymer chains to form network structure. The relationship between the fraction of $T_{2 \mathrm{~s}}$ component and the polymer yield was found to be linear, which gives useful information on this polymerization process in terms of mobility.
\end{abstract}

KEY WORDS Pulsed NMR / Free Induction Decay / Spin-Spin Relaxation Time / Poly(methyl methacrylate) / Bulk Polymerization / Entanglement of Polymer Chains / Network Structure /

So far there have been a number of attempts to observe the polymerization process in a direct manner. ${ }^{1-4}$ Optical microscopy, ${ }^{5} \mathrm{X}$ ray diffraction, ${ }^{6}$ thermal analysis, ${ }^{7}$ and calorimetric method $^{8}$ have been employed for this purpose. Among others, the pulsed nuclear magnetic resonance (NMR) method appears to be particularly suited for studying the polymerization process, because the spin-spin relaxation time is an NMR parameter sensitive to change in molecular mobility which is greatly affected by polymerization. ${ }^{9-12}$

There has been a study on the kinetics of polymerization, which monitors the change in chemical shift of high resolution NMR spectra. ${ }^{13}$ In this paper, the proton spin-spin relaxation time $\left(T_{2}\right)$ of poly(methyl methacrylate) (PMMA) was measured as a function of polymerization time by the pulsed NMR technique. The results were discussed in terms of the yield of polymerization and the molecular weight of the resulting polymer.

\section{EXPERIMENTAL}

Pulsed NMR measurements were carried out with a Bruker P-20 wide line spectrometer operating at a frequency of $19.8 \mathrm{MHz}$. The spin-spin relaxation time was obtained directly from the free induction decay (FID) which follows a $90^{\circ}$ pulse. The width of the $90^{\circ}$ pulse was adjusted from $5 \mu \mathrm{s}$ to $10 \mu \mathrm{s}$. The pulse interval time was $7 \mathrm{~s}$. The dead time of the apparatus was $5 \mu \mathrm{s}$. The FID's were accumulated to improve the signal-tonoise ratio with a Transient Memory $\mathbf{M}$ 100E adapted to an Averager TMC-600 of Kawasaki Electronica Co., Ltd.

Methyl methacrylate (MMA) was distilled twice under reduced pressure. The monomer was sealed with benzoyl peroxide (BPO) 
(BPO : $M M A=1: 136$, molar ratio) in a glass tube $(7 \mathrm{~mm} \phi)$ under reduced pressure. The bulk polymerization was carried out in the NMR probe. Another experiment of the bulk polymerization was also carried out under the same experimental conditions as mentioned above to obtain the yield and molecular weight. The reaction temperature was regulated by an air flow thermostat. After a certain period of reaction, the polymerization was stopped by cooling the sample with liquid nitrogen. Poly(methyl methacrylate) (PMMA) was precipitated by pouring the reaction mixture into a large amount of methanol. The polymer was dried at $80^{\circ} \mathrm{C}$ under reduced pressure and then its weight was measured. The polymer yield was calculated by the following equation;

Polymer yield $(\%)$

$$
=\frac{\text { Weight of produced PMMA }}{\text { Weight of MMA }} \times 100
$$

The average molecular weight was obtained from the intrinsic viscosity measured in acetone at $25^{\circ} \mathrm{C}$ by using Bischoff's equation. ${ }^{14,15}$

\section{RESULTS AND DISCUSSION}

\section{Change of.Free Induction Decay Signal in the Course of Polymerization}

The FID signal of MMA in the early stage of polymerization was apparently of a single exponential type with a long spin-spin relaxation time. On the other hand, the decay curve later became nonexponential. It is obvious that the change in the FID signal shows the progress of polymerization. It has been suggested that the FID curve is exponential for molecules with a shorter correlation time of motion in the liquid state, and that the curve is nonexponential, ${ }^{16}$ i.e., Gaussian, for those with a longer correlation time in the rigid state. As the polymerization proceeds the system changes from the liquid state to the soild or glassy state via the viscous liquid or elastic gel state. We assume that in the course of polymerization the system consists of various components with different $T_{2}$. Thus, the FID curve of the polymerization system $S(\tau)$ can be fitted to the Weibull function ${ }^{12}$

$$
S(\tau)=\sum_{i} S_{0 i} \exp \left[-\left(\tau / T_{2 i}\right)^{E_{i}}\right]
$$

Here, $\tau$ is the time, $E_{i}$ is the Weibull coefficient, $S_{0 i}$, the signal intensity of the $i$-th component at $\tau=0$, and $T_{2 i}$ is the spin-spin relaxation time of the $i$-th component. The fraction of the $j$-th component, $F_{j}$, is given by

$$
F_{j}=S_{0 j} / \sum_{i} S_{0 i}
$$

The Weibull coefficient can be taken on either Gaussian $(E=2)$, exponential $(E=1)$ or intermediate character. ${ }^{9}$ In this experiment, the FID signals were found to be exponential at the early stage of polymerization, and then became the sum of exponential and Gaussian components. In analysis of FID in this experiment, the initial portion of FID was obtained by extrapolation, because it could not be observed for the sake of the dead time. Figure 1 shows the logarithm of the FID of MMA at a reaction time of $17 \mathrm{~h}$ plotted against $\tau$. At times longer than $120 \mu \mathrm{s}$, exponential data fall on a straight line, $L_{1}$,

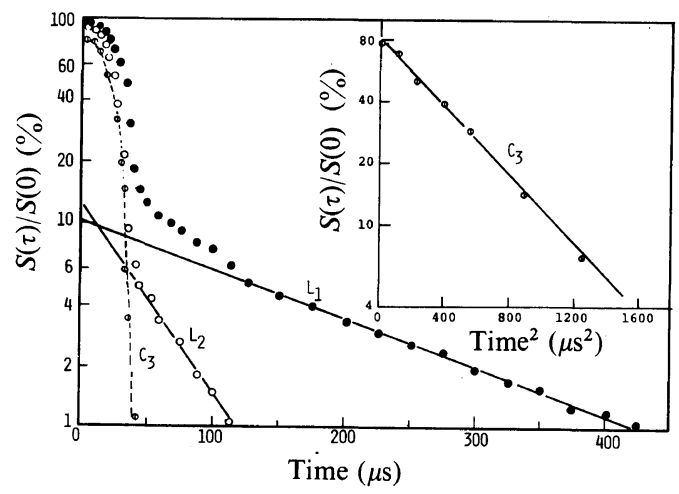

Figure 1. Analysis of FID signal of MMA: reaction time, $17 \mathrm{~h}$; reaction temp, $45^{\circ} \mathrm{C} ; \mathrm{MMA}=1.12 \times 10^{-2}$ $\mathrm{mol} ; \mathrm{BPO}=8.26 \times 10^{-5} \mathrm{~mol}$. 


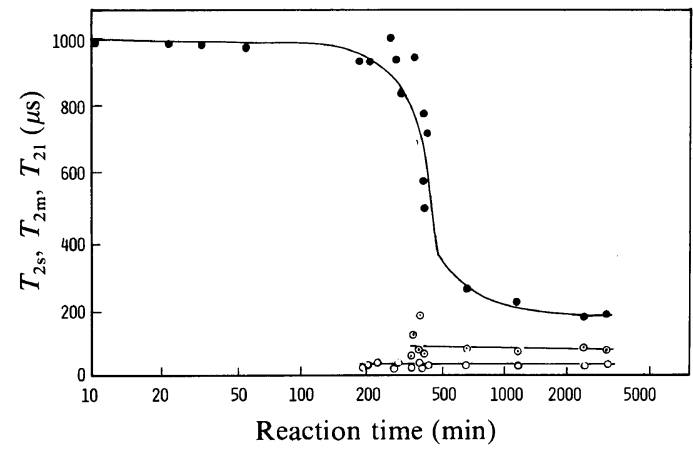

Figure 2. $T_{2 \mathrm{~s}}, T_{2 \mathrm{~m}}$, and $T_{21} v s$. reaction time for MMA at $45^{\circ} \mathrm{C}: \bigcirc, T_{2 \mathrm{~s}} ; \odot, T_{2 \mathrm{~m}} ; \bigcirc, T_{21}$.

giving a longer $T_{21}$. Furthermore, at times $40-120 \mu \mathrm{s}$, the deviation from the line $L_{1}$ (open circles) falls on another straight line $L_{2}$, giving an intermediate $T_{2 \mathrm{~m}}$. At times less than $40 \mu \mathrm{s}$, deviation from $L_{1}$ and $L_{2}$ does not fall on a straight line. This initial portion of the curve $C_{3}$ is shown in the inset of Figure 1 plotted against the square of time. The plot is linear, indicating the curve $C_{3}$ is Gaussian with shorter $T_{2 \mathrm{~s}}$. Thus, we obtain three components with different $T_{2}$. The mass fraction of each component was determined from the intercept of the vertical axis $(\tau=0)$ of each component.

\section{Reaction Time Dependence of Spin-Spin Relaxation Times}

Figure 2 shows the change in spin-spin relaxation time of MMA as a function of the reaction time at $45^{\circ} \mathrm{C}$. Only $T_{21}$ was obtained to the rigid species which arise from the solid reaction time less than $t=200 \mathrm{~min}$. At relatively longer reaction times, the FID was found to be nonexponential, and three different relaxation times were obtained. The longer relaxation time, $T_{21}$, is attributed to mobile species, which are residual monomers and/or low molecular weight polymers. On the other hand, the shortest relaxation time $T_{2 \mathrm{~s}}$ $(15-20 \mu \mathrm{s})$ of the Gaussian decay is attributedto the rigid species which arise from the solid aggregation of the high molecular weight

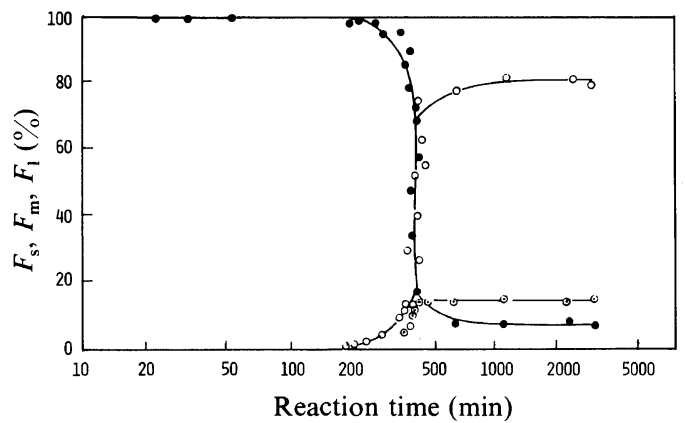

Figure 3. $F_{\mathrm{s}}, F_{\mathrm{m}}$, and $F_{1} v s$. reaction time for MMA at $45^{\circ} \mathrm{C}: \bigcirc, F_{\mathrm{s}} ; \odot, F_{\mathrm{m}} ; \bigcirc, F_{1}$.

polymer. ${ }^{16,17} T_{21}$ remains unchanged at reaction times shorter than $200 \mathrm{~min}$ where it decreases abruptly and component with $T_{2 \mathrm{~s}}$ and $T_{2 \mathrm{~m}}$ begin to be observed. There is no significant change in $T_{2 \mathrm{~m}}$ at reaction times longer than $400 \mathrm{~min}$. The appearance of the component with $T_{2 \mathrm{~m}}$ is somewhat later than that of $T_{2 \mathrm{~s}}$. The appearance of the components with $T_{2 \mathrm{~s}}$ and $T_{2 \mathrm{~m}}$ and decrease of that with $T_{21}$ occur at shorter reaction times as the polymerization temperature rises.

\section{Reaction Time Dependence of Fraction of $T_{2 \mathrm{~s}}$, $T_{2 \mathrm{~m}}$, and $T_{21}$ Components}

Figure 3 shows the change in fraction of $T_{2 \mathrm{~s}}, T_{2 \mathrm{~m}}$, and $T_{21}$ components referred to as $F_{\mathrm{s}}, F_{\mathrm{m}}$, and $F_{1}$, respectively, as a function of reaction time at $45^{\circ} \mathrm{C}$. $F_{\mathrm{s}}$ is zero until the reaction time reaches $200 \mathrm{~min}$, where it abruptly increases to reach about $80 \%$ at longer reaction times. On the other hand, $F_{1}$ decreases abruptly at almost same time as $T_{21}$ shows an abrupt decrease. The abrupt increase in $F_{\mathrm{s}}$ and the abrupt decrease in $F_{1}$ clearly show that the polymerization occurs.

McCall et al. ${ }^{18}$ pointed out in their study on the mixture of polyethylene and paraffin wax that polyethylene is plasticized by the paraffin wax and that the paraffin wax, in turn, is greatly hindered in motion. It is probable that at longer reaction times MMA and low molecular weight PMMA are hindered in motion by high molecular weight PMMA, resulting in 
decrease in $T_{21} . F_{\mathrm{m}}$ is larger than $F_{1}$ at longer reaction times. The meaning of $F_{\mathrm{m}}$ is not clarified yet at present. The $T_{2 \mathrm{~m}}$ component has been also observed for polymer melt. ${ }^{19,20}$

\section{Reaction Time Dependence of $F_{\mathrm{s}}$}

Figure 4 shows the dependence of $F_{\mathrm{s}}$ on the reaction time at various temperatures. $F_{\mathrm{s}}$ at each temperature increases abruptly at a certain reaction time and reaches a saturated value. The abrupt increase occurs at shorter times as the reaction temperature is raised. Figure 5 shows the reaction time dependence of the polymer yield at various temperatures. As well known, in the bulk polymerization of MMA the polymer yield $v s$. reaction time curve shows a sigmoidal increase owing to the "gel effect"21,22 and has a saturated value. This is because the termination reaction is hindered. ${ }^{23}$ The polymer yield curves obtained in this experiment are similar to those of $F_{\mathrm{s}}$. Figure 6 shows the plot of $F_{\mathrm{s}} v s$. the polymer yield at various reaction times and temperatures. A straight line with a slope of $45^{\circ}$ was obtained, although the yield slightly exceeds $F_{\mathrm{s}}$ at polymer yield less than $20 \%$. Table I shows the comparison between $F_{\mathrm{s}}$ and the polymer yield at final stage of polymerization. Taking into the account of experimental errors, $F_{\mathrm{s}}$ is equal to the polymer yield at each temperature. The results show that the pulsed NMR gives us a useful tool for monitoring the polymer yield continuously.

\section{Reaction Time Dependence of Molecular Weight}

Figure 7 shows the dependence of the molecular weight of PMMA on the reaction time. The molecular weight increases in a similar manner as the polymer yield curve. As the temperature is raised, the molecular weight becomes lower. Soh ${ }^{24}$ pointed out that in the bulk polymerization of MMA, the entanglement of polymer chains occurs as the polymer concentration and/or the mo-

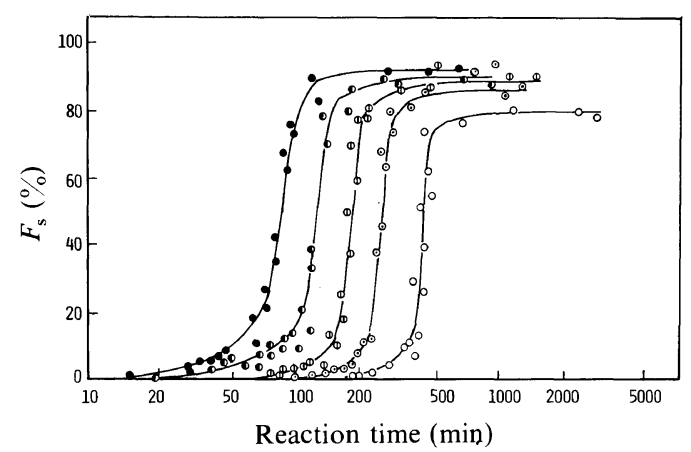

Figure 4. $F_{\mathrm{s}}$ vs. reaction time for $\mathrm{MMA}$ at various temperatures: $\bigcirc, 45^{\circ} \mathrm{C} ; \odot, 50^{\circ} \mathrm{C} ;(1), 55^{\circ} \mathrm{C} ; \mathrm{D}, 60^{\circ} \mathrm{C}$; $65^{\circ} \mathrm{C}$.

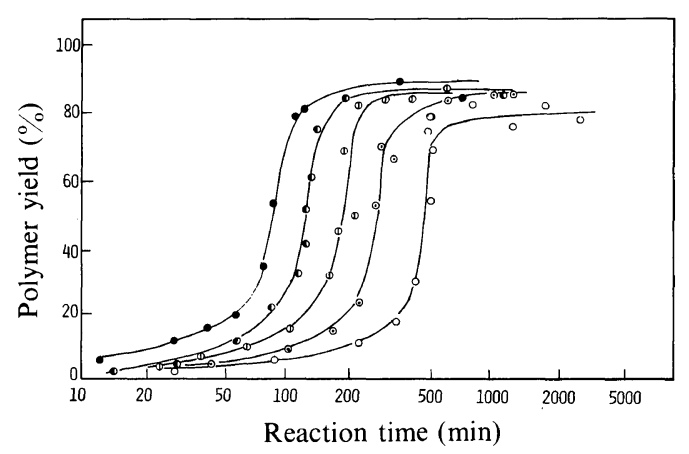

Figure 5. Polymer yield $v s$. reaction time for MMA at various temperatures: $\bigcirc, 45^{\circ} \mathrm{C} ; \odot, 50^{\circ} \mathrm{C}$; (1), $55^{\circ} \mathrm{C}$; $\mathrm{O}$, $60^{\circ} \mathrm{C} ; 0,65^{\circ} \mathrm{C}$.

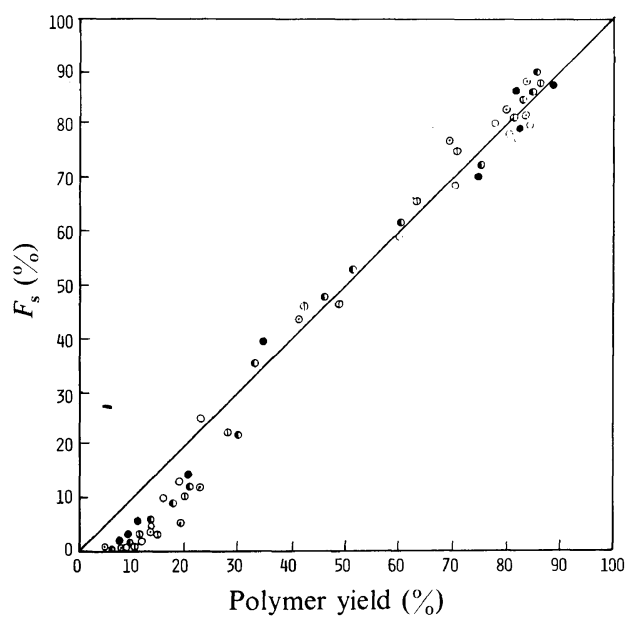

Figure 6. Relationship between $F_{s}$ and polymer yield: $\bigcirc, 45^{\circ} \mathrm{C} ; \odot, 50^{\circ} \mathrm{C} ;(1), 55^{\circ} \mathrm{C} ; \mathrm{O}, 60^{\circ} \mathrm{C} ;-65^{\circ} \mathrm{C}$. 
Table I. Comparison of $F_{\mathrm{s}}$ with polymer yield at various temperatures

\begin{tabular}{crc}
\hline Temp $/{ }^{\circ} \mathrm{C}$ & $F_{\mathrm{s}}^{\mathrm{a}} / \%$ & Polymer yield $^{\mathrm{a}} / \%$ \\
\hline 45 & $81.0 \pm 2.3$ & $80.0 \pm 3.8$ \\
50 & $88.4 \pm 1.7$ & $89.5 \pm 6.7$ \\
55 & $88.9 \pm 1.6$ & $82.9 \pm 1.2$ \\
60 & $90.0 \pm 1.3$ & $85.8 \pm 6.6$ \\
65 & $88.3 \pm 3.7$ & $85.5 \pm 4.1$ \\
\hline
\end{tabular}

a Average of five measurements.

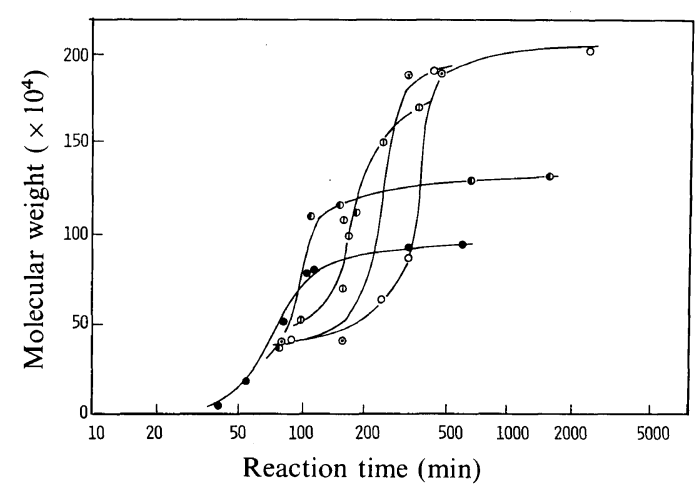

Figure 7. Dependence of molecular weight of PMMA on reaction time: $\bigcirc, 45^{\circ} \mathrm{C} ; \odot, 50^{\circ} \mathrm{C} ;$ (1), $55^{\circ} \mathrm{C} ; \mathrm{O}, 60^{\circ} \mathrm{C}$ : o, $65^{\circ} \mathrm{C}$.

lecular weight increase and that the "gel effect" is closely related to the change of free volume and the occurrence of the entanglement of polymer chains. The critical molecular weight that gives rise to the entanglement effect has been reported to be about $10,000 .{ }^{24}$ From Figures 4 and 7, the molecular weight where the $T_{2 \mathrm{~s}}$ component begins to appear is higher than the critical molecular weight. It could be possible to conclude that the component of $T_{2 \mathrm{~s}}$ corresponds to regions containing entanglements of polymer chains in the reaction mixture. Folland et al. ${ }^{25}$ also pointed out that the spin-spin relaxation time is sensitive to the presence of entanglements when they are present sufficiently for a network structure.

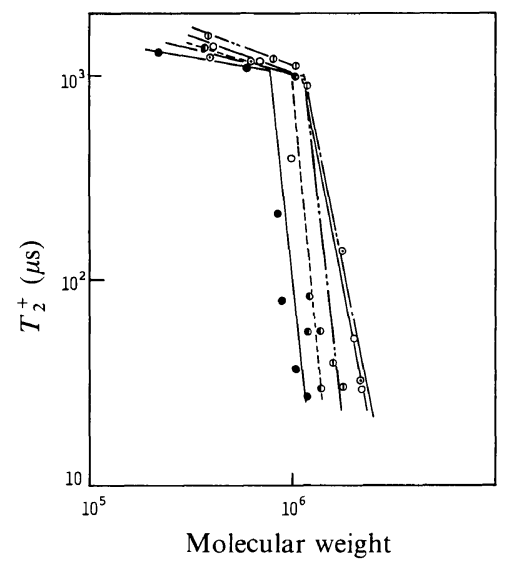

Figure 8. $T_{2}^{+} v s$. molecualr weight of PMMA at various temperatures: $\bigcirc, 45^{\circ} \mathrm{C} ; \odot, 50^{\circ} \mathrm{C} ;$ (1) $55^{\circ} \mathrm{C}$; $\mathrm{O}$, $60^{\circ} \mathrm{C} ; 0,65^{\circ} \mathrm{C}$.

\section{Molecular Weight Dependence of Mean Spin- Spin Relaxation Time}

Relationship between the molecular weight of PMMA and the mean spin-spin relaxation time $\left(T_{2}^{+}\right)$is given in the Figure $8 . T_{2}^{+}$is defined as the time at which the total magnetization decays to $1 / e$ of its initial value. ${ }^{26} T_{2}^{+}$ decreases with increasing molecular weight. The slope of the $T_{2}^{+}$vs. molecular weight plot was found to change at the point where the "gel effect" occurs. This change implies that a drastic decrease in mobility of whole system occurs at this point. Cosgrove et al. ${ }^{27}$ showed that the dependence of $T_{2}$ on the molecular weight for polystyrene solution has a sharp break at a characteristic molecular weight, indicating that the system transforms from relatively viscous solution to entangled network. The abrupt decrease in $T_{2}^{+}$observed in our experiment would be related to the occurrence of a large amount of entanglements of polymer chains which results in the transition of the reaction mixture from solution to immobile glassy soild and in the decrease of free volume of the system. This transition occurs at molecular weight of $c a .0 .8-1.1 \times$ $10^{6}$, independent of temperature. For the PMMA formed in the early stage of polym- 
erization, which has an average molecular weight less than $10,^{6}$ the entanglement exists but only a part of the molecules contributes to affording the $T_{2 \mathrm{~s}}$ components because of the low concentration of the entanglement.

\section{CONCLUSION}

In the bulk polymerization of MMA, three kinds of spin-spin relaxation times $\left(T_{2 \mathrm{~s}}\right.$, $\left.T_{2 \mathrm{~m}}, T_{21}\right)$ were observed, and the fractions $\left(F_{\mathrm{s}}\right.$, $\left.F_{\mathrm{m}}, F_{1}\right)$ of components with three relaxation times were obtained as functions of reaction time and temperature. The $T_{21}$ component arises from molecules that are too small to form the entanglement. The $T_{2 \mathrm{~s}}$ component arises from the motionally hindered region which contains entanglement. In the course of polymerization after the "gel effect" occurs, the value of $F_{\mathrm{s}}$ becomes almost equal to that of the polymer yield. This finding makes it possible to estimate the polymer yield in a nondestructive manner. In this work we showed the time course of polymerization directly and continuously observed by the pulsed NMR. This method is also useful for studying the behavior of the mechanical properties in the polymerization or the crosslinking of rubber, because the spin-spin relaxation time is sensitive to the formation of entanglement of polymer chains (network structure).

Acknowledgements. The author wishes to thank Professor M. Kasagi and Professor M. Nagai of the National Defense Academy and Professor K. Hikichi of Hokkaido University for their critiques and comments.

\section{REFERENCES}

1. C. Chachaty and A. Forchioni, J. Polym. Sci., A-1,
10, 1905 (1972).

2. A. Forchioni and C. Chachaty, J. Polym. Sci., A1, 10, 1923 (1972).

3. Y. Shioji, S. Ohnishi, and I. Nitta, J. Polym. Sci., $A-1,1,3373$ (1963).

4. C. Chachaty, M. Latimier, and A. Forchioni, J. Polym. Sci., Polym. Chem. Ed., 13, 189 (1975).

5. G. Adler, D. Ballantine, and B. Baysal, J. Polym. Sci., 48, 195 (1960).

6. G. Adler and W. Reams, J. Chem. Phys., 32, 1698 (1960).

7. C. Chachaty, M. Magat, and L. Terminassian, J. Polym. Sci., 48, 139 (1960).

8. R. Bensasson and A. Dworkin, C. R. Acad. Sci., (Paris), 256, 4903 (1963).

9. S. Kaufman, J. Polym. Sci., A-2, 9, 829 (1971).

10. H. Tanaka, J. Appl. Polym. Sci., 28, 1707 (1983).

11. R. Folland and A. Charlesby, J. Polym. Sci., Polym. Lett. Ed., 16, 339 (1978).

12. H. Serizawa, M. Ito, T. Kanamoto, K. Tanaka, and A. Nomura, Polym. J., 14, 149 (1982).

13. S. Kobayashi, H. Danda, and T. Saegusa, Bull. Chem. Soc. Jpn., 47, 2699 (1974).

14. J. Bischoff and V. Desreux, Bull. Soc. Chim. Belges, 61, 10 (1952).

15. J. Brandrup and E. M. Immergut, Ed., "Polymer Handbook," 2nd ed, Interscience, New York, N. Y., 1975.

16. I. Kamel and A. Charlesby, J. Polym. Sci., Polym. Phys. Ed., 19, 803 (1981).

17. R. Folland and A. Charlesby, Polymer, 20, 211 (1979).

18. D. W. McCall, D. C. Douglass, and E. W. Anderson, J. Polym. Sci., 59, 301 (1962).

19. H. Tanaka, J. Appl. Polym. Sci., 27, 2197 (1982).

20. D. W. McCall, D. C. Douglass, and D. R. Falcone, J. Phys. Chem., 71, 998 (1967).

21. G. V. Schulz and G. Harborth, Makromol. Chem., 1, 106 (1947).

22. E. Trommsdorff, H. Köhle, and P. Lagally, Makromol. Chem., 1, 169 (1948).

23. M. S. Matheson, E. E. Auer, E. B. Bevilacqua, and E. J. Hart, J. Am. Chem. Soc., 71, 497 (1949).

24. S. K. Soh and D. C. Sundberg, J. Polym. Sci., Polym. Chem. Ed., 20, 1345 (1982).

25. R. Folland, J. H. Steven, and A. Charlesby, J. Polym. Sci., Polym. Phys. Ed., 16, 1041 (1978).

26. R. A. Assink, J. Appl. Polym. Sci., 26, 3689 (1981).

27. T. Cosgrove and R. F. Warren, Polymer, 18, 255 (1977). 